\title{
The effect of nanoparticle size on thermal diffusivity of gold nano-fluid measured using thermal lens technique
}

\section{E. Shahriari}

esmaeil.phy@gmail.com

\section{W. M. Mat Yunus}

\section{R. Zamiri}

Department of Physics, Faculty of Science, Shahrekord University, P.O. Box 115, Shahrekord, Iran

Department of physics, Universiti Putra Malaysia, 43400UPM Serdang, Malaysia

A dual beam mode-mismatched thermal lens method has been employed to investigate the dependence of thermal diffusivity of gold nanofluid on nanoparticles sizes. The samples were prepared at various sizes by utilizing the gamma radiation method. In the dual beam mode-mismatched thermal lens a diode laser $(532 \mathrm{~nm}$ ) was used as an excitation beam and a He-Ne laser with the beam output at $632.8 \mathrm{~nm}$ was used as a probe beam. Thermal diffusivity of gold nano-fluid increased with the increasing particle sizes ranging from 10.4 to $29.6 \mathrm{~nm}$. [DOI: http://dx.doi.org/10.2971/jeos.2013.13026]

Keywords: Thermal diffusivity, thermal lens, nanoparticle

\section{INTRODUCTION}

Metal nanoparticles are important class of photonic materials due to the local field enhancement under the resonance plasmon generation condition which leads to enhancement of various light-induced linear and nonlinear optical processes within nanoscopic volume of the media surrounding the nanoparticles $[1,2]$. They also have received considerable attention recently because of their potential as high performance heat transfer fluids in automotive and electronic cooling [3, 4] and in microchannel heat sinks [5]. Metal nanoparticles have been proposed as targeted thermal agents for use in medical therapies and drug delivery [6]-[8], and could extend the precision of thermal effects below cellular dimensions [9].

The enhancement in the thermal diffusivity values of the solvents such as ethanol, water, ethylene glycol and culture medium by the presence of gold nanoparticles have been studied [10,11]. For example, with $0.033 \mathrm{mmol} \mathrm{Au}$ in $50 \mathrm{ml}$ of ethanol, water and ethylene glycol made the thermal diffusivity increased by $0.7,7.0$ and $8.4 \%$, respectively. It was also reported that with 4 vol. \% of nanoparticles of $\mathrm{CuO}(23.6 \mathrm{~nm}$ diameter) in ethylene glycol made the thermal diffusivity increased by $22 \%$ [12]. This phenomenon could be due to the electrostatic interaction between the cationic solution and the negatively charged Au nanoparticle. Thus hot electron generated by laser excitation may rapidly release thermal energy through electron-phonon scattering process [13].

The concentration dependence of the thermal diffusivity of nanofluids has been widely investigated, for example Jimenez Perez et al. [14] reported the increases thermal diffusivity of R6G dye mixed with gold nanoparticles was due to the increasing of Au nanoparticle concentration. However, very few published articles reporting the effect of nanoparticles size on the thermal transport properties of nanofluids. In the present work, we report thermal diffusivity measurements of gold nanofluids at five different sizes of gold nanoparticles in a polymer matrix using a double beam thermal lens technique and all samples were prepared using $\gamma$-radiation method.

\section{EXPERIMENTAL}

For preparing the Au-PVA nanoparticles samples, hydrogen tetrachloroaurate (III) hydrate, premion (metals basis), ( $\mathrm{HAuCl}_{4} .3 \mathrm{H}_{2} \mathrm{O}$ with $99.999 \%$ purity), $2.5 \mathrm{~g}$ polyvinyl alcohol, PVA, $1 \mathrm{ml}$ isopropanol were used for preparing Au nanofluid sample. The PVA and isopropanol were used as a colloidal stabilizer and hydroxyl radical scavenger respectively. The PVA solutions were made by dissolving PVA powder in $50 \mathrm{ml}$ deionized water at room temperature. The solution was magnetically stirred for 2 hours and was bubbled with nitrogen gas $(99.5 \%)$ to remove oxygen. $\gamma$-radiation $\left({ }^{60} \mathrm{Co}-\right.$ rays) was used as an effective tool for polymerization process and reducing agent same as procedure reported in our previous paper [15]. Hydrogen tetrachloroaurate (III) hydrate, $\mathrm{HAuCl}_{4} \cdot 3 \mathrm{H}_{2} \mathrm{O}$ at weight of $5 \mathrm{mg}$ was added into PVA solution to produce $\mathrm{Au}$ nano-fluid sample. The $\mathrm{Au}$ ion concentration of $2.943 \times 10^{-4} \mathrm{M}$ in PVP solution were then irradiated with $\gamma$-radiation at dose of $10,30,40,50$ and $70 \mathrm{kGy}$ to produce various nanoparticle sizes ranging from $10.4 \mathrm{~nm}$ to $29.6 \mathrm{~nm}$. In this process, $\gamma$-irradiation produces hydrated electrons that reduce the gold ions to gold atoms, which then aggregated to certain particle sizes in the solution. The average diameter of Au nanoparticles was measured using nanophox machine (Sympatec GmbH, D-38678) and listed in Table 1. We determined the shape of the particles and their polydispersity 


\begin{tabular}{|c|c|c|c|c|}
\hline Nano-Fluid samples & Average particle size $(\mathrm{nm})$ & $\mathrm{t}_{\mathcal{c}}\left(10^{-3} \mathrm{~s}\right)$ & $\theta$ & $\mathrm{D}\left(10^{-3} \mathrm{~cm}^{2} / \mathrm{s}\right)$ \\
\hline S1 & 10.4 & $7.70 \pm 0.24$ & $1.014 \pm 0.016$ & $1.534 \pm 0.484$ \\
\hline S2 & 15.0 & $6.46 \pm 0.16$ & $1.242 \pm 0.017$ & $1.803 \pm 0.457$ \\
\hline S3 & 17.0 & $5.69 \pm 0.22$ & $1.593 \pm 0.091$ & $2.047 \pm 0.812$ \\
\hline S4 & 20.5 & $4.55 \pm 0.09$ & $1.211 \pm 0.011$ & $2.510 \pm 0.535$ \\
\hline S5 & 29.6 & $4.03 \pm 0.11$ & $1.391 \pm 0.014$ & $2.891 \pm 0.081$ \\
\hline
\end{tabular}

TABLE 1 Thermal diffusivity of Au nano-fluid measured at concentration of $2.943 \times 10^{-4} \mathrm{M}$.

for a few samples via transmission electron microscopy. We found that the particles distribution in the solution is in uniform shape.

The thermal lens (TL) spectrometry has been developed as a technique for characterization of absorption in optical samples $[16,17]$. The thermal lens effect is caused by deposition of heat via a non-radiative decay process after laser energy has been absorbed by the sample. In this situation, a transverse temperature gradient is established, and due to the temperature coefficient of refractive index $(\mathrm{dn} / \mathrm{dT})$ a refractive index gradient is produced, creating a lens-like optical element, the so-called thermal lens (TL). The propagation of a TEMoo Gaussian laser beam, either the excitation laser beam itself or a probe beam through the TL will be affected, resulting in a spreading of the beam and a reduction in the intensity at the beam center (when $\mathrm{dn} / \mathrm{dT}$ is negative), or focusing of the beam and increasing in its intensity at the beam centre (when $\mathrm{dn} / \mathrm{dT}$ is positive). By measuring the beam centre intensity in the far field, the thermo-optical properties of the sample can be obtained [18]-[20]. The time-resolved method permits measurements of the development of the thermal lens in a short time. In this case, the thermal diffusivity can be determined [21].

Figure 1 shows the schematic diagram of a dual beam mode mismatched thermal lens experiment used in the present measurement. The experiments were performed using a $532 \mathrm{~nm}$ laser beam from Laser-diode (Coherent Compass SDL-532$150 \mathrm{~T})$ as an excited beam and a He-Ne laser with wavelength of $632.8 \mathrm{~nm}$ as a probe beam. The excitation beam was focused to a small spot using a lens of $21 \mathrm{~cm}$ focal length on $2 \mathrm{~mm}$ quartz optical cell. The transmitted probe beam in the far field passed through the aperture and was recorded by a detector D. The excitation laser beam waist $\omega_{0}$ at the focus length was measured to be $68.3 \mu \mathrm{m}$ and the Rayleigh length was found to satisfy the basic criteria of the thermal lens experiment. The linear absorption spectra for samples were measured using UV-Vis spectrophotometer (Shimadzu-UV1650PC).

\section{THEORY}

The TL signal is defined as the normalized change of the transmission of the probe beam, centered at the beam axis and located at a distance much larger than the pump Rayleigh parameter. Shen et al in 1995 have derived as expression for the signal using a diffraction approximation for Gaussian beams, which is given by: [21]

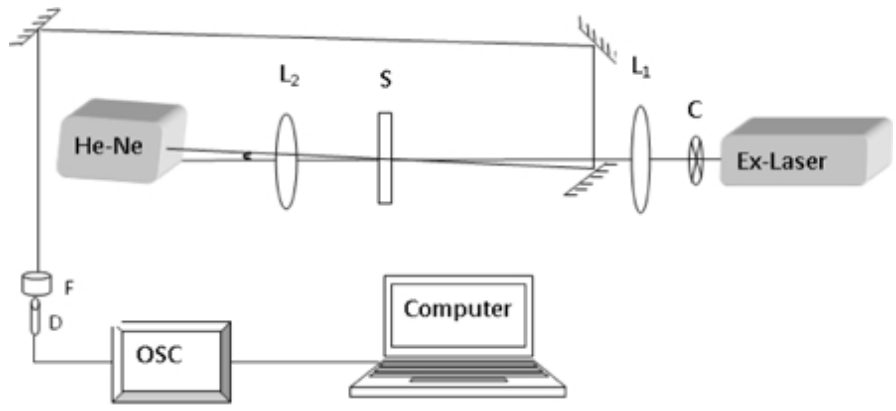

FIG. 1 Schematic experimental setup of Thermal Lens; $C$, Chopper; $L_{1}, L_{2}$, Lenses; $S$, Sample; F, Filter; D, Detector.

$$
\begin{aligned}
& I(z, t) \\
= & I_{0}\left(1-\theta \tan ^{-1}\left(\frac{2 m V}{\left[(1+2 m)^{2}+V^{2}\right] \frac{t_{c}}{2 t}+1+2 m+V^{2}}\right)\right)^{2}
\end{aligned}
$$

Where

$$
\begin{gathered}
V=\frac{z_{1}}{z_{c}} ; \quad m=\left(\frac{w_{p}}{w_{e}}\right)^{2} ; \quad t_{c}=\frac{w_{e}^{2}}{4 D^{\prime}}, \\
\theta=\frac{P_{e} \cdot \alpha \cdot l}{\kappa \lambda_{P}} \frac{d s}{d T}
\end{gathered}
$$

in which $\lambda_{p}$ is the wavelength of the probe beam, D and kare the thermal diffusivity and thermal conductivity, $P_{e}$ is the excitation beam power, $\alpha$ is the absorption coefficient, and $l$ is the sample thickness, $z_{c}=w_{o}^{2} / \lambda$ is the confocal distance $(\mathrm{cm})$, $w_{o}$ is the probe beam waist radius, $w_{p}$ and $w_{e}$ are the probe and the excitation beam radii at sample, respectively, $z_{1}$ is the distance of laser beam waist to sample, $t_{c}$ is the characteristic thermal time constant. In Eq. (1), $I(t)$ is the temporal dependence of the probe beam intensity at the detector, $I_{0}$ is the initial value of, $I(t)$. The $d s / d$ Tis the temperature coefficient of the optical path length change, at the probe beam wavelength, which is written as [16, 23]:

$$
\frac{d s}{d T}=(n-1)(1+v) \alpha_{T}+\frac{d n}{d T}
$$

where $\alpha_{T}$ is the sample linear thermal expansion coefficient, $v$ is the Poisson ratio, and $d n / d T$ is the temperature coefficient of its refractive index.

\section{RESULTS AND DISCUSSION}

Figure 2 shows the UV-Vis absorption spectra of fluids containing $\mathrm{Au}$ nanoparticles prepared at a concentration of $2.943 \times 10^{-4} \mathrm{M}$ at different particle sizes. An absorption peak 


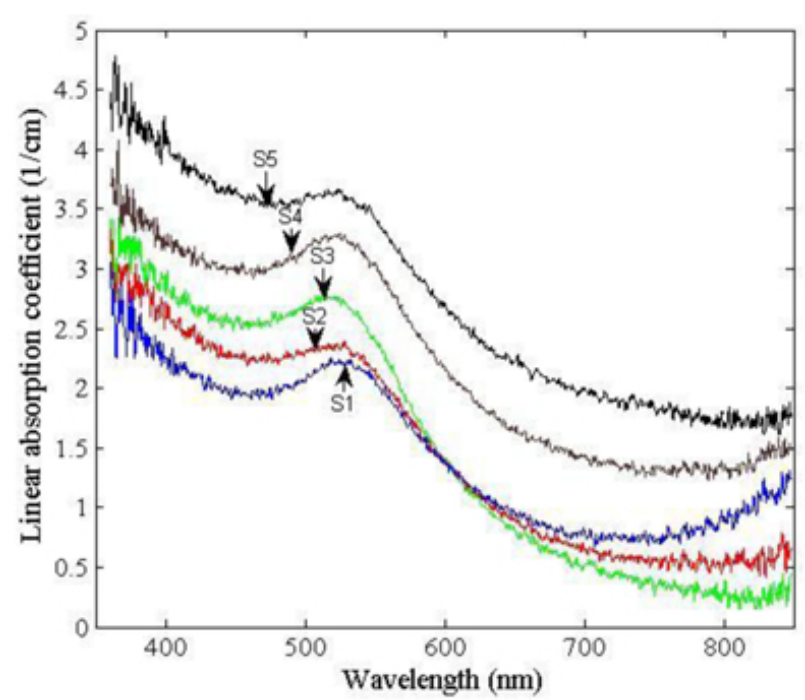

FIG. 2 Optical Absorption spectra of fluids containing Au nanoparticles prepared at different sizes of metal ions.
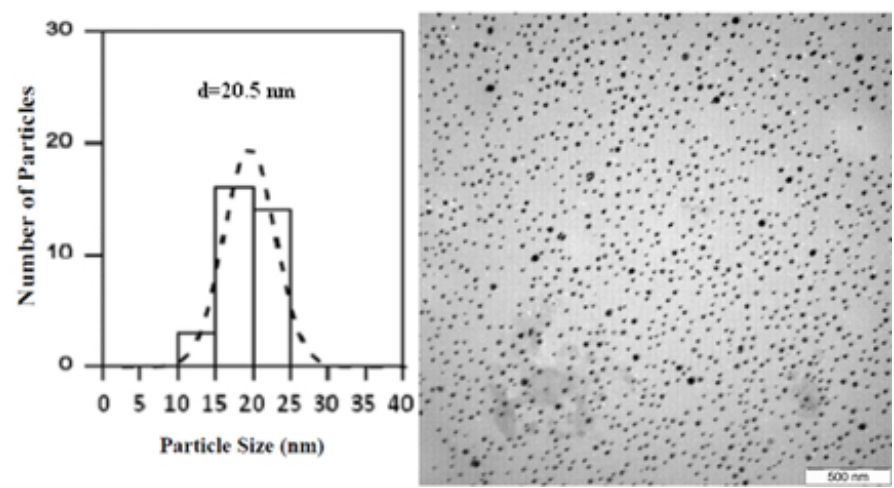

FIG. 3 TEM images and particles size histograms of Au particles with a nominal size of $20.5 \mathrm{~nm}$.

at $525 \mathrm{~nm}$ was revealed in these fluids, which is generally assigned to the surface plasmon resonance (SPR) of $\mathrm{Au}$ nanoparticles [24]. From this figure, we observed that a decrease in sizes of Au nanoparticles in the solution shift the optical absorption peak towards higher wavelengths. Figure 3 displays a typical TEM image showing particle distributions of the $20.5 \mathrm{~nm}$ Au nanoparticles and shape of the particle.

Figure 4 shows a typical TL signal as a function of time for $\mathrm{Au}$ nanoparticles dispersed in poly vinyl alcohol-distilled water irradiated with $30 \mathrm{kGy}$. The theoretical curves in the Figure 4 calculated using Eq. (1) agrees very well with experimental data, which give the thermal diffusivity value for the sample as $2.510 \times 10^{-3} \mathrm{~cm}^{2} / \mathrm{s}$. Thus by plotting the thermal diffusivity value as a function of nanoparticles sizes it shows the thermal diffusivity of the sample increases with the increasing of particle sizes [25]. This behavior is displayed in Figure 5 and agrees with the reasons highlighted by previous researchers such as, when the dimensions of particles become in order of phonon mean free path, phonon scattering effect become smaller as a result increase the thermal diffusivity [26]. When there is a different rate of phonon transport in two materials it creates a thermal resistance at the solid-liquid interface and this can cause increasing thermal diffusivity value of the medium due to the increasing of particle size [27]. Furthermore, for a poor

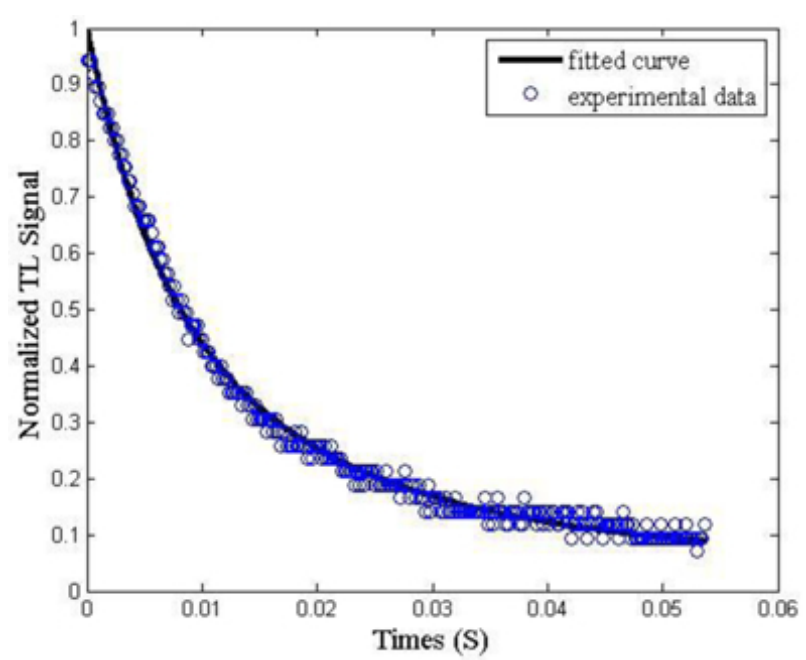

FIG. 4 Typical time evolution of the Thermal Lens signal for Au nanofluid (S4) Symbol (0) represents the experimental data and solid line is the best fitting line to Eq. (1).

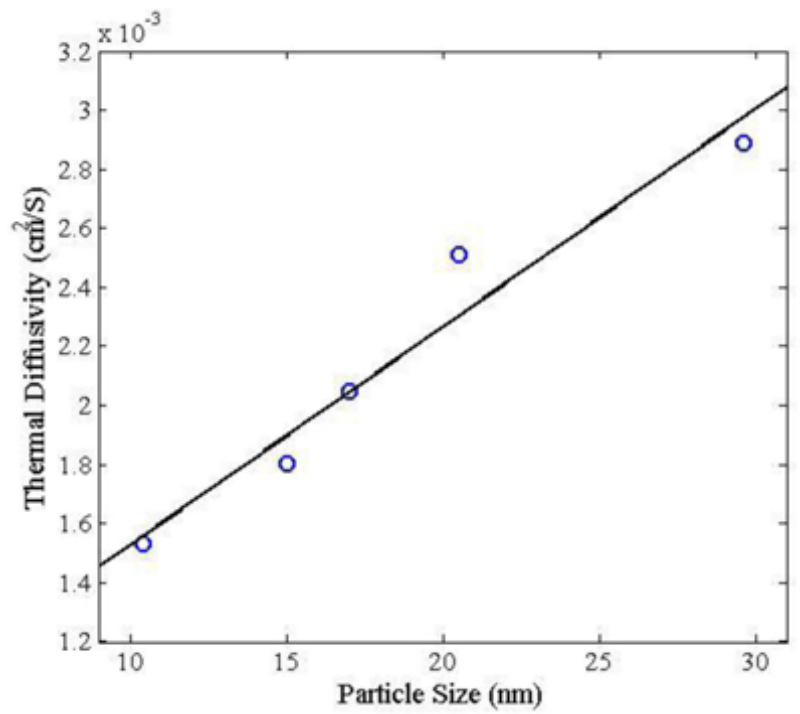

FIG. 5 Variation of thermal diffusivity of Au nano-fluid versus the particles size.

contact between the materials and phonon dynamics can also decrease the thermal diffusivity of nanofluids as particle size decreases [28].

\section{ACKNOWLEDGEMENTS}

We gratefully acknowledge the Department of Physics, UPM for providing the research facilities to enable us to carry out this research. One of the authors (W. M. Mat Yunus) would also like to acknowledge the MOSTI for the financial support through Fundamental research grant (01-11-08664FR/5523664).

\section{References}

[1] D. Compton, L. Cornish, E. Van der Lingen, "The third order nonlinear optical properties of gold nanoparticles in glasses," Cold Bull. 36, 51-58 (2003).

[2] P. N. Prasad, Nanophotonics (Wiley, New York, 2004). 
[3] S. E. Maiga, C. T. Nguyen, and N. Galanis, "Heat transfer enhancement in turbulent tube flow using $\mathrm{Al}_{2} \mathrm{O}_{3}$ nanoparticle suspension," Int J. Numer. Method. H.16, 275-292 (2006).

[4] D. S. Wen, and W. Ding, "Natural convective heat transfer of suspensions of titanium dioxide nanoparticles (Nanofluids)," IEEE. T. Nanotechnol.5, 220-227 (2006).

[5] S. P. Jang, and S. U. S. Choi, "Cooling performance of a microchannel heat sink with nanofluids," Appl. Therm. Eng. 26, 2457-2463 (2006).

[6] K. H. Schifferli, J. J. Scwartz, A. T. Santos, S. G. Zhang, and J. M. Jacobson, "Remote electronic control of DNA hybridization through inductive coupling to an attached metal nanocrystal antenna," Nature 415, 152-156 (2002).

[7] C. Loo, A. Lin, L. Hirsch, M. H. Lee, J. Barton, N. Halas, J. West, and R. Drezck, "Technol Nanoshell-Enabled Photonics-based Imaging and Therapy of Cancer," Cancer. Treat. 3, 33-40 (2004).

[8] D. P. O'Neal, L. R. Hirsch, N. J. Halas, J. D. Payne, and J. L. West, "Photo-thermal Tumor Ablation in Mice Using Near Infraredabsorbing Nanoparticles," Cancer. Lett. 209, 171-176 (2004).

[9] G. Huttmann, and R. Birngruber, "On the possibility of highprecision photothermal microeffects and the measurement of fast thermal denaturation of proteins," IEEE J. Sel. Top. Quant. 5, 954-962 (1999).

[10] J. L. J. Perez, R. G. Fuentes, J. F. S. Ramirez, and A. C. Orea, "Study of gold nanoparticles effect on thermal diffusivity of nanofluids based on various solvents by using thermal lens spectroscopy," Eur. Phys. J.-Spec. Top. 153, 159-161 (2008).

[11] J. L. J. Perez, R. G. Fuentes, E. M. Alvarad, E. R. Gallegos, A. C. Orea, J. T. Cordova, and J. G. M. Alvarez, "Enhancement of the thermal transport in a culture medium with Au nanoparticles," Appl. Surf. Sci. 255, 701-702 (2008).

[12] Q. Xue, and W. M. Xu, "A Model of Thermal Conductivity of Nanofluids with Interfacial Shells," Mater. Chem. Phys. 90, 298-301 (2005)

[13] C. V. Bindhu, S. S. Harilal, V. P. N. Nampoori, and C. P. G. Vallabhan, "Solvent effect on absolute fluorescence quantum yield of rhodamine $6 \mathrm{C}$ determined using transient thermal lens technique," Mod. Phys. Lett. B 13, 563-576 (1999).

[14] J. L. J. Perez, J. F. S. Ramırez, R. G. Fuentes, A. C. Orea, and J. L. H. Perez, "Enhanced of the R6C Thermal Diffusivity on Aggregated Small Gold Particles," Braz. J. Phys. 36, 1025-1028 (2006).

[15] E. Shahriari, W. M. M. Yunus, K. Naghavi, and Z. A. Talib, "Effect of concentration and particle size on nonlinearity of Au nano-fluid prepared by $\gamma\left({ }^{60} \mathrm{Co}\right)$ radiation," Opt. Commun. 283, 1929-1932 (2010).
[16] S. E. Bialkowski, Photothermal Spectroscopy Methods for Chemical Analysis (Wiley, New York, 1996).

[17] J. Shen, M. L. Baesso, and R. D. Snook, "Three-dimensional model for $\mathrm{cw}$ laser-induced mode-mismatched dual-beam thermal lens spectrometry and time-resolved measurements of thin film samples," J. Appl. Phys. 75, 3738-3748 (1994).

[18] T. Imasaka, K. Sakaki, and N. Ishibashi, "Determination of iron (II) with 2-nitroso-5-diethylaminophenol by thermal lens spectrophotometry using a semiconductor laser as a light source," Anal. Chim. Acta. 243, 109-113 (1991).

[19] J. Shen, and R. D. Snook, "Thermal lens measurement of absolute quantum yields using quenched fluorescent samples as references," Chem. Phys. Lett. 155, 583-586 (1989).

[20] J. M. Harris, and N. J. Dovichi, "Thermal lens calorimetry," Anal. Chem. 52, 695-706 (1980).

[21] J. Shen, R. D. Lowe, and R. D. Snook, "Two-beam Thermal Lens Spectrometer for Ultra-trace Analysis," Chem. Phys. 18, 403-408 (1998).

[22] J. Shen, A. J. Soroka, and R. D. Snook, "A model for cw laser induced mode-mismatched dual-beam thermal lens spectrometry based on probe beam profile image detection," J. Appl. Phys. 78 , 700-708 (1995).

[23] M. Sparks, "Optical distortion by heated windows in high power laser systems," J. Appl. Phys. 42, 5029-5046 (1971).

[24] J. Turkevich, "Colloidal Gold Part II: Colour, Coagulation, Adhesion, Alloying and Catalytic Properties," Gold Bull. 18, 125-131 (1985).

[25] J. L. Jiménez-Pérez, J. F. Sánchez-Ramírez , D. Cornejo-Monroy, R. Gutierrez-Fuentes, J. A. Pescador Rojas, A. Cruz-Orea, M. A. Algatti, et al., "Photothermal Study of Two Different Nanofluids Containing $\mathrm{SiO}_{2}$ and $\mathrm{TiO}_{2}$ Semiconductor Nanoparticles," Int. J. Thermophys. 33, 69 (2012).

[26] D. G. Cahill, W. K. Ford, and K. E. Goodson, "Nanoscale thermal transport," J. Appl. Phys. 93, 793-818 (2003).

[27] C. W. Nan, R. Birringer, and D. R. Clarke, "Effective thermal conductivity of particulate composites with interfacial thermal resistance," J. Appl. Phys. 81, 6692-6699 (1997).

[28] Z. B. Ge, D. G. Cahill, and P. V. Braun, "Thermal conductance of hydrophilic and hydrophobic interfaces," Phys. Rev. Lett. 96, 186101-186104 (2006). 